\title{
A novel automatic procedure for measuring ocular counterrolling: A computeranalytical method to determine the eye's roll angle while subjects work on perceptual tasks
}

\author{
URS BUCHER \\ University of Zurich, Zurich, Switzerland \\ FRIEDRICH HEITGER \\ ETH Zurich, Zurich, Switzerland \\ and \\ FRED MAST and NORBERT BISCHOF \\ University of Zurich, Zurich, Switzerland
}

\begin{abstract}
In investigations of the perception of space, the consideration of ocular counterrolling-the movement of the eye around its visual axis in response to body movement-is crucially important. The angle of this movement must be known in order for one to determine the precise retinal coordinates of a distal object. Following transformation, this stimulus serves as a reliable cue for visual direction. The otolith organs provide information about body tilt and are responsible for ocular counterrolling. A novel, noninvasive method to measure ocular counterrolling, based on the cross-correlation of digitized video pictures of the eyes, is presented. The resolution attained was $\leq 0.1^{\circ}$. The computer analysis is fully automatic and fast, and it can be performed while subjects work on perceptual tasks. No direct access to the eyeballs is required. Data from 4 subjects showing the counterrolling profile in various body positions are presented.
\end{abstract}

We are primarily interested in spatial orientation, and particularly in vertical perception. To investigate this phenomenon further, we bring our human subjects into various body positions in a dark environment, where they are presented with a luminous line. The subjects are requested to set this luminous line to the apparent vertical.

A system-analytical approach, as initiated by Bischof and Scheerer (1970), envisages the problem of the perception of the vertical as a compensatory process. The angle of body tilt, it has been argued, has to be compensated for by central-nervous processing (Bischof, 1966).

The position of the line with respect to the body position allows us to calculate the angular deviation of the subjective vertical from the gravity vector (see Figure 1). In order to determine the precise compensatory angle used

The authors are grateful for the assistance of Walter Schmid and Henri Gossweiler, who built most of the technical equipment. We thank Christopher Pryce for critical reading of the manuscript and his helpful suggestions. Special thanks are also due to the subjects who patiently took part in the experiments. Anyone who is interested in applying the presented algorithm should contact the authors. The programs are written in FORTRAN 77 using specific routines to access the video digitizer. We plan to adapt the software to Apple Macintosh computers with the appropriate hardware for video digitizing. Requests for reprints should be sent to Urs Bucher, University of Zurich, Department of Psychology, Biomathematical section, Attenhoferstrasse 9, $\mathrm{CH}-8032$ Zurich, Switzerland.

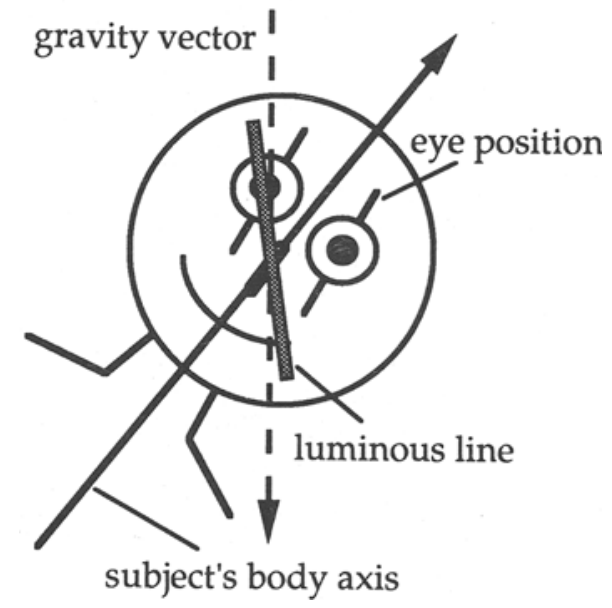

Figure 1. Schematic representation of the experimental task. Subjects are instructed to set a luminous line to vertical. The eyes rotate opposite to the direction of body tilt.

to transform retinal signals, we need information about the alignment of the line with respect to the position of the eyes (Bischof, 1974; Bucher, 1988). Such alignment is, however, affected by the degree to which the eyes rotate, given a particular amount of body rotation. As the head is tilted sideways, the eyes are counterrolled around 
the visual axis. This eye movement is called ocular counterrolling (OCR).

In this article, we present a novel, highly accurate, and noninvasive procedure for measuring OCR. Our procedure is based on the quantitative analysis of videotaped pictures of the eyes. The single frames are digitized to compare the iris pattern of a specific video frame with a reference frame taken in the upright body position. This comparison is carried out in various runs by crosscorrelation.

\section{METHOD}

\section{Apparatus}

Our apparatus allowed us to tilt human subjects and the entire apparatus described below into any desired body position with respect to gravity. Secured by a helmet and a neck support, the subject looked through binoculars. A stereoscopic presentation of the visual stimulus (luminous line) required firm stabilization of the head. This was provided by individual dental impressions on a removable bite-board. The bite-board was also necessitated by the on-board camera macro lens, which only had a narrow focal range. The position of the eyes relative to the binoculars was not critical, but it was necessary to stabilize the head at a specific distance from them. We suggest that the most convenient position may be the visual axis normal to the head's frontal plane.

We monitored each eye independently on video. Using semireflecting mirrors, the visual stimulus was displayed in the optical axis of the camera, which coincided with the optical axis of the subject. The eye to be recorded was chosen by selective illumination (see Figure 2). The light source consisted of an infrared diode directed toward the eyeball. For control reasons, the digitized video signal was displayed on a screen, allowing the experimenter to optimize recording quality for the subsequent analysis. To avoid recording out of focus, the lens of the camera could be adjusted by remote control during ongoing experiments. A time/date signal was added to the video frames containing the eye pictures and was recorded on videotape so that it could be synchronized with the perceptual data collected.

\section{Analysis}

Single frames of the video pictures were processed by image analysis of the iris pattern. ${ }^{1}$ Each frame was converted by a video digitizer into a $512 \times 512$ pixel image

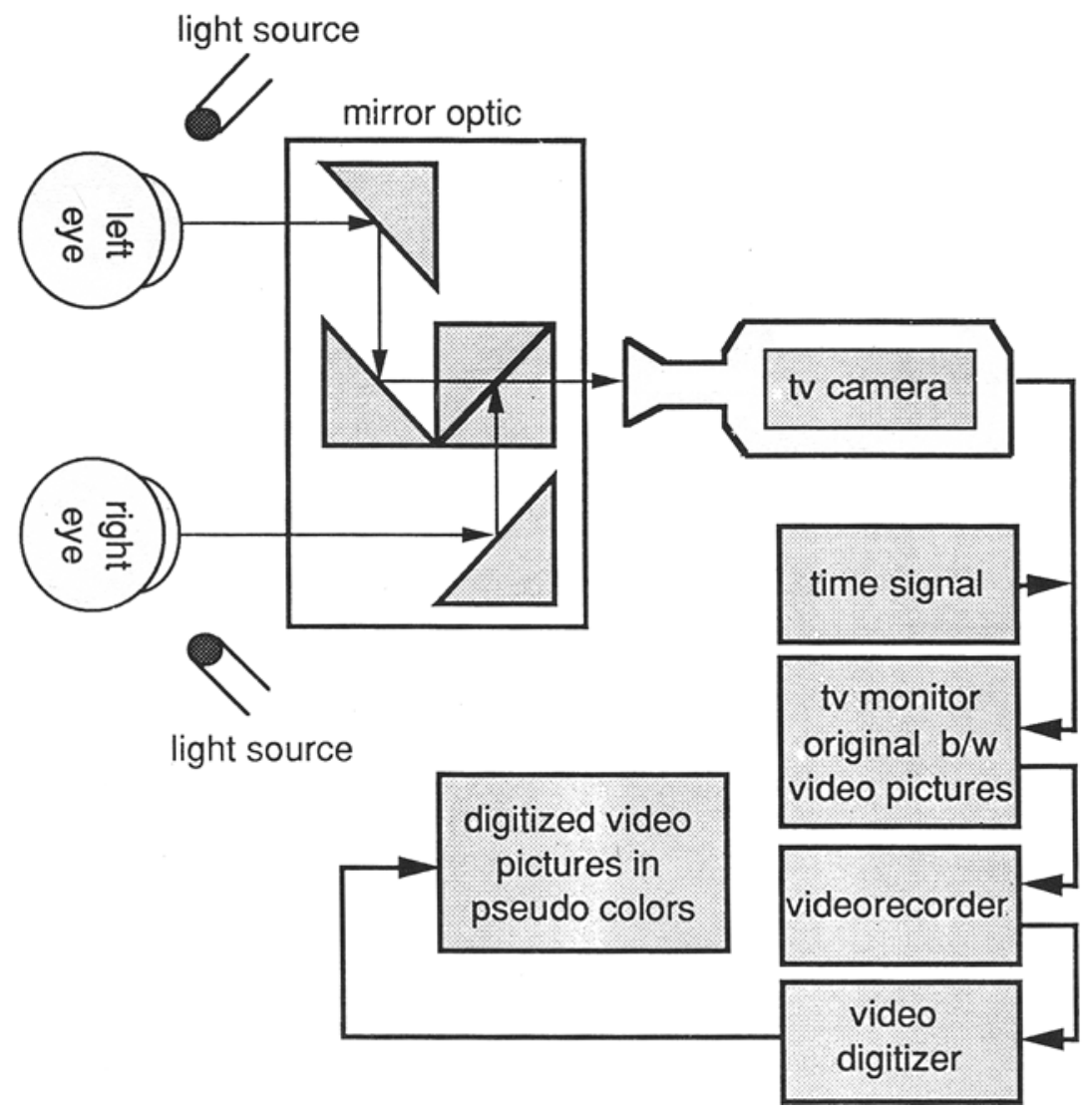

Figure 2. The flow chart shows the recording of the pictures of the eyes. Through the mirror optics the camera points to both eyes, which can be illuminated selectively. 


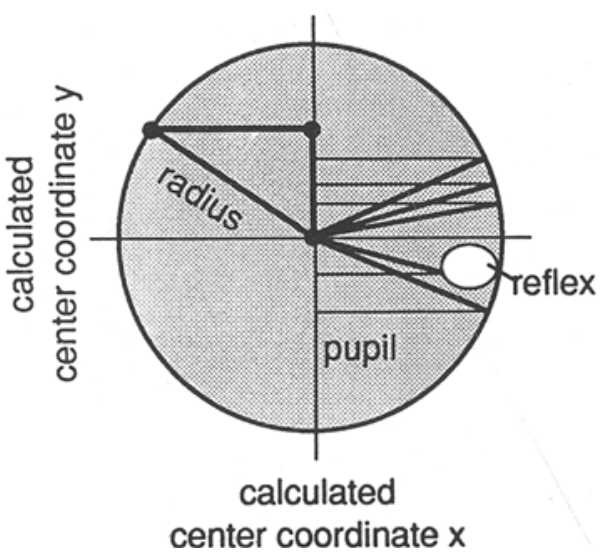

Figure 3. Method to determine pupil size. The different radii obtained are stored in a histogram. The most represented size in the histogram is selected as the final radius.

with 4-bit resolution. Thus, the image was compressed to 16 gray values. This information was later subjected to computer analysis.

Our method of determining OCR angles was based on an automatic analysis of the iris pattern. In particular, we cross-correlated parts of the iris pattern with reference data that were taken in the upright head position (defined by the so-called "Frankfurt horizontal," which cuts through the cranium at the lowest points of the inferior orbital ridges and the middle of the external auditory meatii). The iris pattern varies considerably with pupil size. As a consequence, a mismatch in pupil size diminishes the quality of cross-correlation and thereby the accuracy of OCR measurement. To overcome this problem, we took reference images in the upright position for 10 different pupil sizes by varying the ambient illumination spanning the naturally occurring range during each experiment. The center and radius of the pupil were determined by the following procedures: Starting from the center of the image, the pupil boundaries were separately determined in the $x$ and $y$ directions. Simple thresholding has proven to be sufficient to separate the dark pupil from the rim of the iris pattern surrounding it. The $x$ coordinate of the pupil center was determined by the midpoint between a line connecting left and right pupil borders. Histogram methods were used to determine the final $x$-coordinate of the pupil center. The frequency of each $x$-coordinate of the midpoints was calculated, and the modal value was used. In comparison with simple averaging, this histogram analysis is much more robust in relation to local disturbances (e.g., reflections or specularities). The $y$-coordinate of the pupil center was determined accordingly.

A sample radius of the pupil could be calculated on a simple pythagorean basis, as is illustrated in Figure 3. The pupil radius was determined at different locations. The final radius was again determined by histogram methods: The frequency of occurrence of the different radii were stored, and the modal radius was used for data analysis.

In the further analysis, the radius was used to select the appropriate reference data set. The iris pattern of the experimental image was subsequently scanned on 10 con-

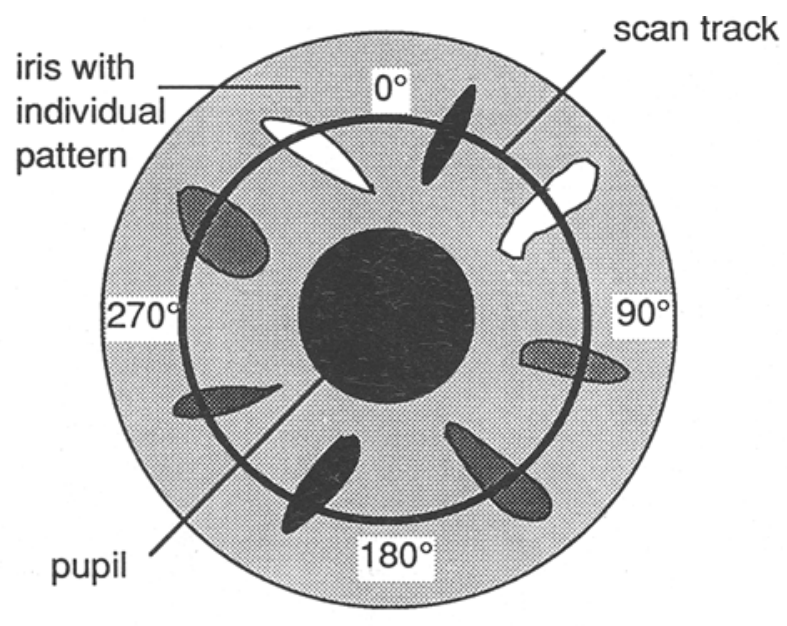

sequence of scanned grey values (pattern string)

for comparison with reference pattern

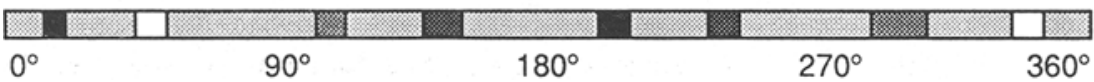

Figure 4. Digitized iris pattern with one scan track. The resulting pattern string of gray values is shown below. 
centric circles whose radii corresponded to those of the reference data set (see Figure 4). The pattern strings of the reference images and those taken during the experiments were cross-correlated. The location of the peak of the cross-correlation function was taken as their phase difference and corresponded directly to the OCR angle.

Figure 4 demonstrates the scan procedure. The circle around the pupil represents one scan track. Each scan circle consists of 720 data points sampled in $0.5^{\circ}$ intervals. The pattern string below shows the sequence of gray values at corresponding positions of the $360^{\circ}$ scan track.

Figures 5 and 6 explain the cross-correlation procedure. Figure 5a shows schematically the pattern of a reference data set, and Figure $5 \mathrm{~b}$ is an example of a corresponding data set taken in a tilted body position. The scan track from $0^{\circ}$ to $360^{\circ}$ is represented on the abscissa. The ordinate displays the 4 different gray levels used (for purposes of simplicity, the actual number of 16 steps has been reduced to 4). Figure 6 shows the cross-correlation function of both input patterns.
Figure 6 shows the resulting cross-correlation value as a function of the difference between the phase of the reference scan and the phase of the scan on the eyes recorded experimentally. The peak of the curve represents the minimum phase difference; that is, the two patterns showed their maximum correspondence. The cross-correlation procedure was repeated over an angular interval of $-15^{\circ}$ to $+15^{\circ}$ in a total of 60 steps of $0.5^{\circ}$ each. As an option, a loop may be aborted at a correlation coefficient of 1 . To increase the robustness of the OCR measurement, the same procedure was run for 10 different tracks with varying radii on the same video frame. We then calculated the mean and standard error of OCR angles received from these 10 measurements. Angles with standard errors larger than $0.25^{\circ}$ were not accepted. High standard errors were mainly associated with bad video quality or artifacts introduced by the subjects (e.g., eye blinks). For each body position tested, we ran this procedure for at least 10 independent single frames with each eye. With this statistical method, the OCR could be measured with an overall

\section{a) pattern of reference frame}

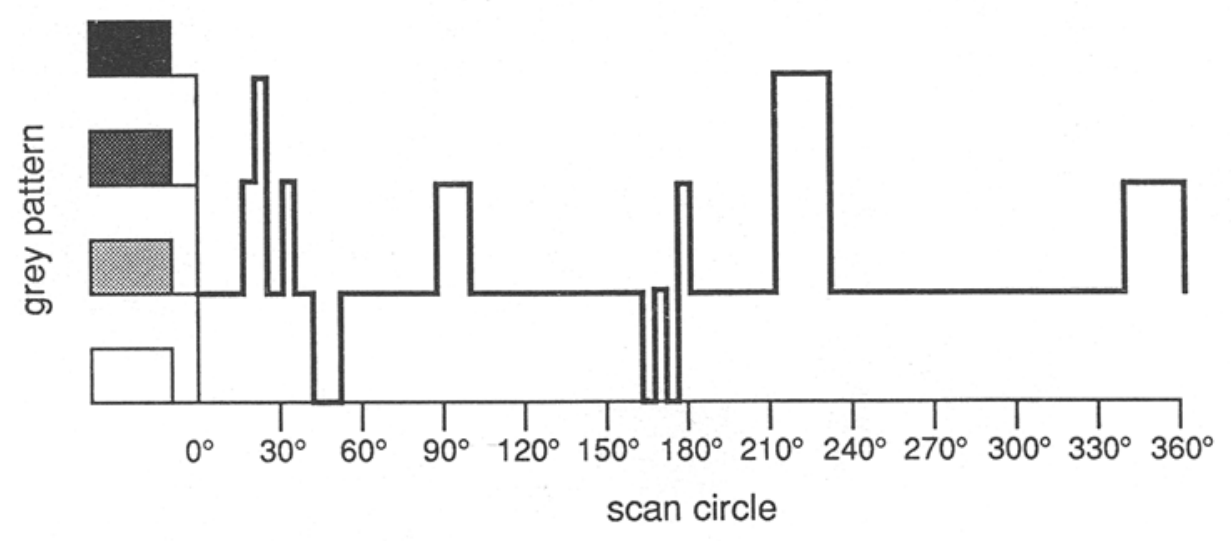

\section{b) pattern of frame to be analyzed}

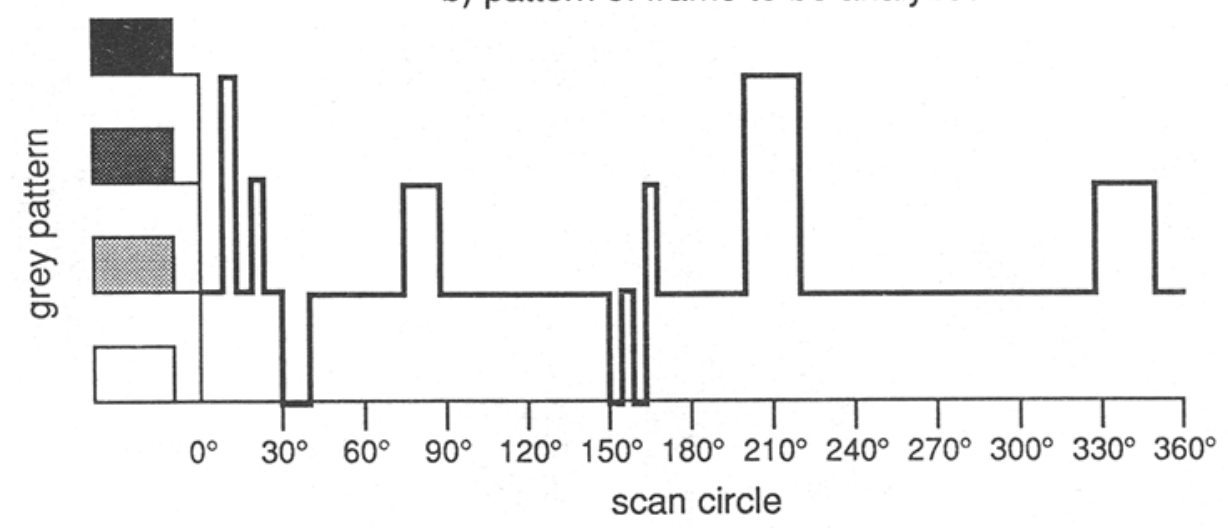

Figure 5. The pattern strings of a reference frame and a frame to be analyzed. The extent of the shift of the two strings along the $\boldsymbol{x}$-axis is determined by cross-correlation. 


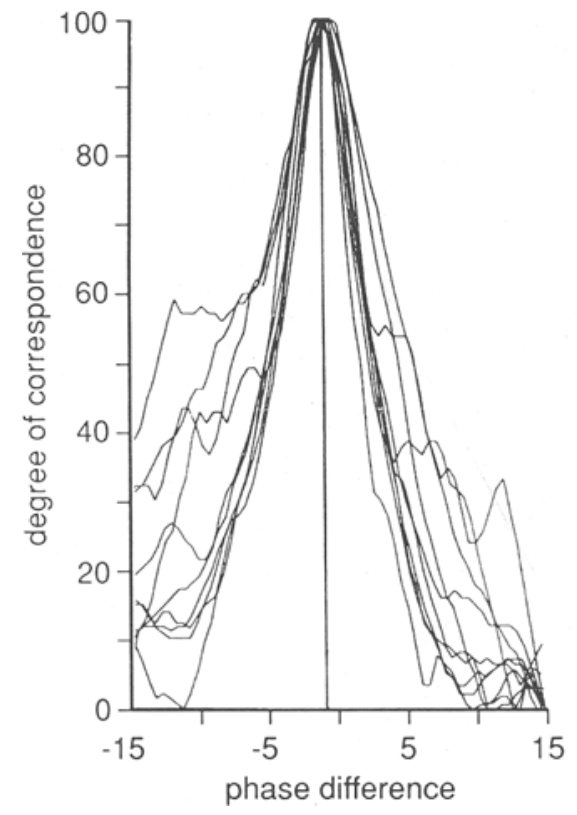

Figure 6. Resulting cross-correlation profile of 10 different pattern strings to determine one $\mathrm{OCR}$ angle. The peaks show the optimal correspondence with the reference frame.

accuracy of $0.1^{\circ}$. This is below the angular variation produced by the permanent flickering to which the eyeball is subjected.

\section{Experimental Setting}

An application of the method described above is presented below. The data were recorded in lateral body positions. The roll angle was varied in intervals of $30^{\circ}$. During one experimental session, our subjects went sequentially through 7 different positions from $0^{\circ}$ to $180^{\circ}$ (clockwise, with respect to subject) or from $0^{\circ}$ to $-180^{\circ}$ (counterclockwise). After a time of 5-10 min in each body position, during which the subject set a luminous line to the apparent vertical, each eye was recorded on video for $10 \mathrm{sec}$.

\section{Subjects}

Four subjects took part in our experiments ( 2 men, 2 women, aged between 25 and 40 years). Their state of health was checked by standard medical testing prior to their inclusion in the study.

\section{RESULTS}

Figure 7 shows the OCR angle recorded from the right and the left eyes in 4 subjects. The angle is the mean value of the 10 different video frames taken in each body position. All subjects showed the characteristic sinusoidal modulation of OCR. Large differences in OCR amplitude as well as in the standard errors were observed between subjects. Standard errors varied from $0.04^{\circ}$ to $0.76^{\circ}$ (mean $0.21^{\circ}$ ): Subject M.A. had relatively low values (a maximum of only $0.18^{\circ}$ ); Subject G.O. had relatively high values (most deviations were above $0.20^{\circ}$ ).

\section{DISCUSSION}

In this study, we have presented a method for measuring OCR during testing of subjects for the perceived vertical. Our method allows us to determine the OCR angle without any uncomfortable manipulation of the subjects' eyeballs. A continuous infrared light source, rather than visible strobe lighting, is used to obtain the necessary video images. Thus, no visible light interferes while the subjects are performing perceptual tasks.

The time required to process one single video frame was less than $30 \mathrm{sec}$, including grabbing the frame, scanning the iris, and calculating the cross-correlations. ${ }^{2}$ It should be possible to perform the analysis on-line during experiments in which the subject is occupied with perceptual tasks. On the other hand, postexperimental data analysis allows us to base measurements on any desired number of different video frames. This provides a means of testing the reliability of the results. The off-line version is recommended in the case of individuals possessing an iris structure with low contrast. Such eyes provide only a small amount of variation in the resulting scan pattern, and this pattern is important for a reliable crosscorrelation. Such pictures tend to have large standard errors between the 10 different scans. Our experience has shown that we can compensate for this flat scan pattern by increasing the number of video frames analyzed to find enough frames with a tolerable standard error.

The method, in which an actual frame is compared with a reference frame, enables the processing of any pupil size within a predefined range. This is important, since it is well known that the pupil size varies not only with light intensity but also with psychological state.

In our experimental setting, relatively large standard errors were highly correlated with bad image quality. High standard errors could be identified as being primarily a problem of illumination. Gravitational forces acting on the eyeballs change their position and, consequently, the entrance angle of the light source. In addition, the glittering of the eye caused by increased tearing reduces the contrast of the iris pattern.

The OCR values presented correspond to those shown in other studies (e.g., Bischof \& Scheerer, 1970; Udo De Haes, 1970). The peaks in OCR should, according to the literature, occur at $60^{\circ}$ body tilt. In six out of eight cases, we observed peaks at tilts of $\pm 90^{\circ}$. This, however, is not contradictory. We made measurements in discrete $30^{\circ}$ steps. The differences between OCR at $90^{\circ}$ and at $60^{\circ}$ are not significant, and, presumably, the real peaks are between body tilts of $60^{\circ}$ and $90^{\circ}$. As in other experiments, we found a slight asymmetric trend that depended on the side of body roll. Although the general shape of the curves remained sinusoidal, we measured remarkable interindividual differences with respect to amplitude. This 


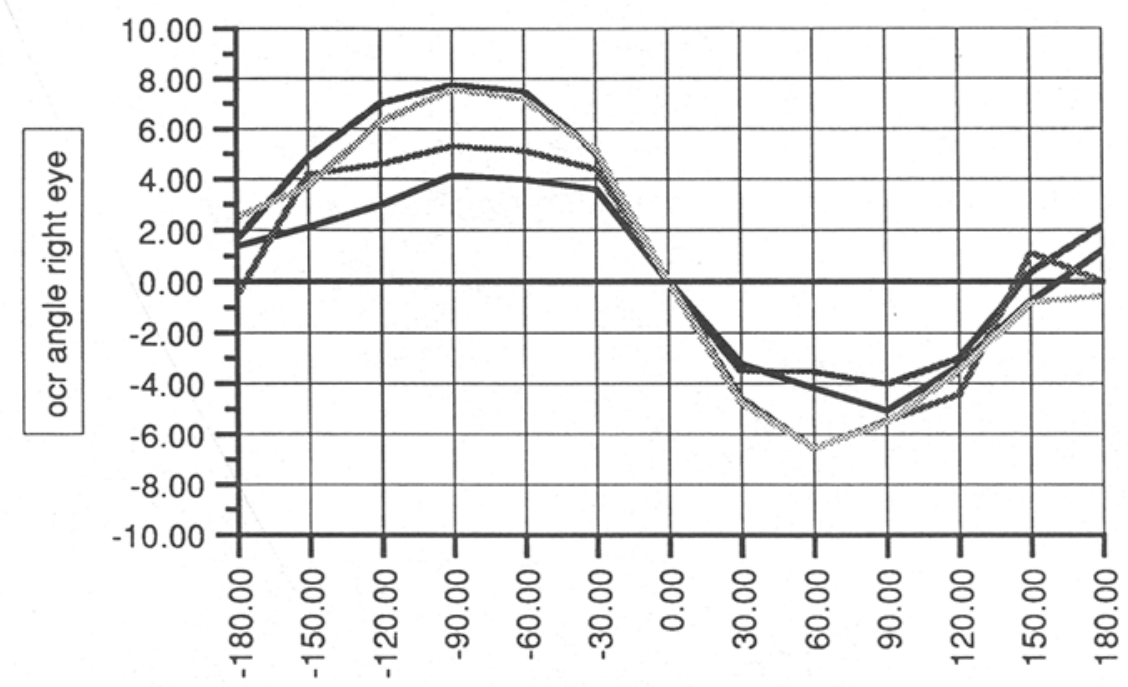

body position (roll angle)

\section{Subject MA - Subject GO - Subject CA - Subject MD}

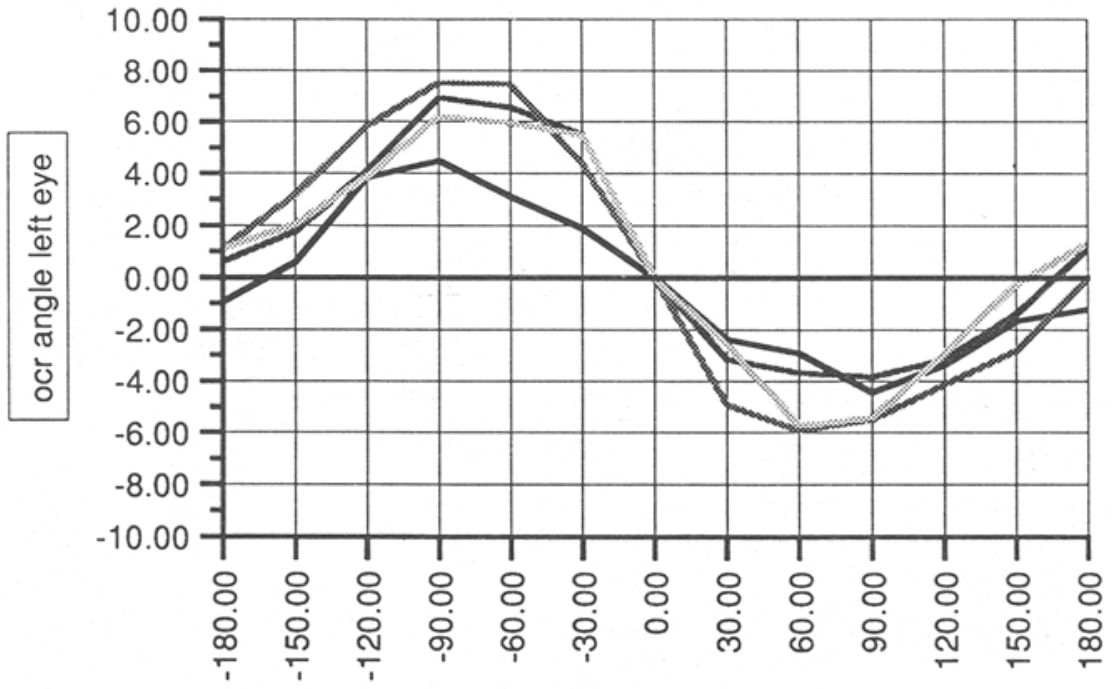

body position (roll angle)

Figure 7. OCR angles of 4 subjects. The body position was varied between $-180^{\circ}$ and $180^{\circ}$. Standard errors, though not given, were moderate (see text). 
sinusoidal characteristic could be accounted for by the afferent signals of the utricles (Fernandez \& Goldberg, 1976), which are considered to control the OCR.

The results of other experiments carried out with an increased number of different body positions in threedimensional space (Bucher, Mast, \& Bischof 1990) agree with the characteristics of the OCR profile reported here.

\section{REFERENCES}

Bischof, N. (1966). Psychophysik der Raumwahrnehmung. In W. Metzger \& H. Erke (Eds.), Handbuch der Psychologie: Vol. I, Pt. 1. Bewusstsein und Wahrnehmung (pp. 307-408). Göttingen: Verlag für Psychologie.

BischoF, N. (1974). Optic-vestibular orientation to the vertical. In H. H. Kornhuber (Ed.), Handbook of sensory physiology: Vol. 6 , Pt. 2. Vestibular system (pp. 155-190). Heidelberg: Springer.

Bischof, N., \& Scheerer, E. (1970). Systemanalyse der optischvestibulären Interaktion bei der Wahrnehmung der Vertikalen. Psychologische Forschung, 34, 99-181.

BUCHER, U. (1988). Orthogonalitat des subjektiven Wahrmehmungsraumes unter Ausschluss visueller Stimuli zur Raumorientierung. Unpublished doctoral dissertation, University of Zurich.
Bucher, U., Mast, F., \& Bischof, N. (1990). An analysis of ocular counterrolling in response to body positions in three-dimensional space. Manuscript submitted for publication.

Fernandez, C., \& Goldberg, J. (1976). Physiology of peripheral neurons innervating otolith organs of the squirrel monkey: I. Response to static tilts and to long-duration centrifugal force. Joumal of Neurophysiology, 39, 970-984.

Udo De HaEs, H. A. (1970). Stability of apparent vertical and ocular countertorsion as a function of lateral tilt. Perception \& Psychophysics, 8, 137-142.

\section{NOTES}

1. The analysis is primarily based on an algorithm that was developed by F. Heitger as part of the Swiss National Science Foundation Project No. 1.559-0.82/1.82.

2. The processing was done on a PDP-11/73 computer and a MATROX QVAF-512 video digitizer board. Processing time could be much improved by omitting visual control of the scans during the analysis. The whole program could easily be transferred to other machines (e.g., an Apple Macintosh computer equipped with an appropriate video digitizer and a FORTRAN 77 compiler).

(Manuscript received April 5, 1990; revision accepted for publication August 30, 1990.) 